\title{
A Narrative Identity Perspective on Mechanisms of Change in Imagery Rescripting
}

\author{
Soljana Çili $^{1,2 *}$ and Lusia Stopa ${ }^{2}$ \\ ${ }^{1}$ London College of Fashion, University of the Arts London, London, United Kingdom, ${ }^{2}$ School of Psychology, University of \\ Southampton, Southampton, United Kingdom
}

Keywords: imagery rescripting, intrusive images, mechanisms of change, narrative identity, self-defining memories

\section{INTRODUCTION}

Imagery rescripting (ImRs) is increasingly used in cognitive-behavioral therapy (CBT) to change beliefs and meanings about the self associated with negative and traumatic memories. It is quintessentially an imagery intervention that targets the self and autobiographical memory (AM); however, to date most of the research into its effectiveness has focused on symptom alleviation. The mechanisms of change remain unclear. In this article, we outline a narrative identity model of change in ImRs and note the value of the narrative identity literature in helping us understand memory-focused therapeutic interventions.

\section{OPEN ACCESS}

Edited by:

Jorge Javier Ricarte,

University of Castilla-La

Mancha, Spain

Reviewed by:

Maria Verónica Jimeno Jimenez,

University of Castilla-La

Mancha, Spain

*Correspondence:

Soljana Çill

S.Cili@fashion.arts.ac.uk

Specialty section:

This article was submitted to

Psychopathology,

a section of the journal

Frontiers in Psychiatry

Received: 30 November 2020 Accepted: 22 November 2021 Published: 16 December 2021

Citation:

Çili S and Stopa L (2021) A Narrative Identity Perspective on Mechanisms of Change in Imagery Rescripting. Front. Psychiatry 12:636071.

doi: 10.3389/fpsyt.2021.636071

\section{IMRS AS A TOOL FOR ADDRESSING INTRUSIVE MENTAL IMAGES IN CLINICAL DISORDERS}

ImRs describes a family of techniques in which patients recall and then imaginatively change a disturbing image/memory. The aims of this imagery manipulation can include any of the following: introducing a new perspective (e.g., seeing a childhood memory of sexual abuse from an adult perspective), contextualizing or adding information to the memory that was unavailable at the time (e.g., understanding that one survived despite believing that one would die during the trauma), seeing how the memory contributed to negative beliefs or experiences of the self, realizing how the memory contributes to distressing intrusive images, and activating a more understanding and compassionate attitude toward the self. The methods range from unstructured approaches exploring interventions such as bringing the adult self into the memory to structured approaches such as Arntz and Weertman's (1) three-stage protocol. The latter involves activating the memory in Stage 1. In Stage 2, the patient relives the memory again from the perspective of the adult self and is encouraged to intervene in any way s/he wishes. In Stage 3, the patient goes through the memory from the younger self perspective with the adult present so that the younger self can ask for any help that s/he needs but was not provided by the adult in Stage 2. Some applications of ImRs [e.g., (2)] use cognitive restructuring before embarking on ImRs that follows Arntz and Weertman's protocol.

Evidence suggests that ImRs is an effective intervention. A recent meta-analysis showed that it produced symptom alleviation and a significant reduction in the vividness of memory-related intrusive images and associated distress across a range of disorders (3). The minority of studies which looked at beliefs associated with the rescripted memories (the "encapsulated beliefs") showed reductions following ImRs. Subsequently published research has examined the effects of ImRs in binge eating disorder (4), obsessive-compulsive disorder (5-7), major depressive disorder $(8,9)$, illness anxiety disorder $(10)$, social anxiety disorder $[\operatorname{SAD} ;(11,12)]$, borderline personality disorder (13), psychosis (14), and voice hearers (15). Overall, this research supports previous findings on 
ImRs outcomes. Moritz et al. (8) also found improvements in self-esteem following a self-help ImRs intervention, although other researchers [e.g., (15)] failed to find this effect. Taken together, the existing studies suggest that memory and selfprocesses are central to ImRs. Current explanations of change focus on these processes.

\section{EXISTING EXPLANATIONS OF THE MECHANISMS OF CHANGE IN IMRS}

Explanations of change in ImRs exist at different levels. The most basic level focuses on associative learning processes and places the change in the meaning attached to the targeted memories/images at the heart of its therapeutic effects $(1,16-$ 18). In this explanation, ImRs allows the reactivation of the distressing memories/images (the unconditioned stimuli), their emotional processing, and subsequent re-evaluation before they are reconsolidated. As a result of the re-evaluation, future activations of the rescripted unconditioned stimuli produce a new conditioned response (a diminished emotional response) which reflects the new meaning.

Higher-level explanations of change focus on the link between the change in meaning or encapsulated beliefs and the self. Mancini and Mancini (19) propose that ImRs contributes to meta-emotional changes. Seeing the experience from the perspective of the adult self allows the person to empathize with the younger self and initiates a change in the perception of the younger self's negative emotions. Specifically, earlier suffering may be perceived as "legitimate, adequate, and deserving of care" [(19), p. 3], resulting in acceptance of negative emotions instead of seeing them as problematic. This reappraisal may lead to reduced reactivity to these emotions, changes in selfrepresentations, and symptom alleviation.

In a precursor to the model we describe below, Çili et al. (20) explained the effects of ImRs by drawing on the self-memory system (SMS) model $(21,22)$ and Brewin's (23) retrieval competition hypothesis. The SMS model proposes that the SMS initiates a search for a relevant self-defining memory when there is a shift in environmental demands that requires a response. The activation of this memory is accompanied by an affective response and the activation of a working self comprising goals and self-images. The retrieval competition hypothesis argues that cognitive-behavioral interventions like ImRs may contextualize aversive memories and make new or existing positive self-representations more likely to win the retrieval competition against negative self-representations (23, 24). Combining these two explanations, we proposed that ImRs facilitates the integration of aversive self-defining memories with individuals' other AMs by changing their meaning, and this may reduce the salience and accessibility of the memories and related working selves (20). As a result, the benign working selves gain a retrieval advantage in situations that would have previously favored negative working selves. According to our account, ImRs modifies the impact of the aversive memory on the working self and the reduced distress results from benign working self activation. In fact, Norton and Abbott (25) suggest that ImRs may reduce access to negative self-imagery and associated meanings and create new positive meanings or images. Our study showed that 1 week after rescripting a non-clinical sample rated their aversive memory as less negative, less distressing, and less important for their sense of self, reporting reduced post-retrieval state negative affect and higher state self-esteem and positive affect (20).

Despite encouraging evidence supporting the proposals above, the exact mechanisms operating in ImRs are yet to be uncovered. We argue that taking a broader picture of the memory-self relationship is critical to advancing the field and argue that the narrative identity literature provides a promising framework for understanding change in ImRs. Our expanded model is presented below.

\section{A NARRATIVE IDENTITY MODEL OF CHANGE IN IMRS}

We propose that understanding mechanisms of change in ImRs requires an understanding of AM functions and personality. First, AM serves at least 3 main functions: directive (guiding behavior, emotion, and cognition), social (developing and nurturing interpersonal relationships), and self (constructing and maintaining a sense of self) [e.g., $(21,22,26-28)]$. These functions can be adaptive or maladaptive (29) and this may vary across the lifespan (30). Second, McAdams [e.g., (31-33)] proposes that personality consists of three layers: dispositional traits; characteristic adaptations (e.g., goals, values, hopes, fears); and the life story or narrative identity, a constantly changing narrative about who one is, was, and may become which gives individuals a sense of unity, self-continuity, and direction in life. The life story allows individuals to integrate their emotions, cognitions, and behaviors so they can pursue their long-term goals $(31,32,34)$. Germane to our model is evidence suggesting that, overall, life stories or AMs are associated with low levels of well-being or symptoms of psychological disorders when they are described with a low sense of agency (e.g., autonomy) and/or communion (interpersonal connection), are characterized by negative affect, and reflect contamination (shifts from a positive to a negative outcome) or negative connections between the memory and the self (e.g., "This experience shows that I am a failure") [for reviews, see $(35,36)$; see also (37)].

We also propose that our understanding of ImRs may benefit from literature on the self and personality outcomes of therapy. This literature is relatively limited. A systematic review on self changes in CBT for SAD, for example, concluded that CBT produced significant reductions in negative self-related thoughts and beliefs and significant increases in positive ones (38). These changes often predicted and/or mediated therapeutic outcomes. Similarly, changes in maladaptive self-beliefs during CBT have been found to predict subsequent changes in the severity of SAD symptoms (39). When it comes to personality, evidence suggests that psychological interventions may lead to changes in some traits. In particular, neuroticism and introversion may decrease following therapy (40-43). The sense of agency expressed in memory narratives, on the other hand, may increase $(44,45)$. 


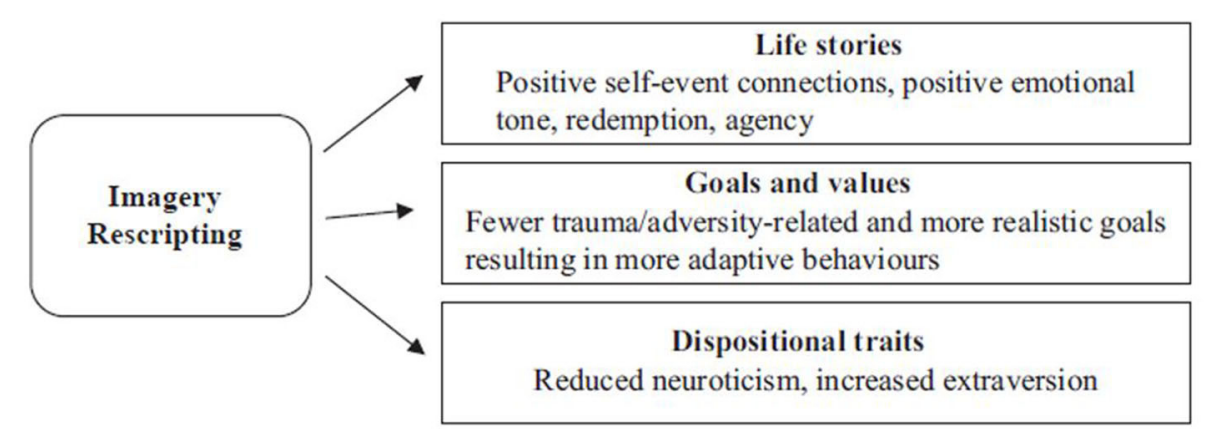

FIGURE 1 | Narrative identity model of change in imagery rescripting. Adapted from Çili and Stopa (36). Copyright 2019 by S. Çili and L. Stopa. Reproduced by permission of Taylor and Francis Group, LLC, a division of Informa plc.

This may result from interventions such as CBT contributing to increased fulfillment of psychological needs, including autonomy and mastery (46).

Based on the existing literature, our model (36) proposes that interventions like ImRs promote change at each personality layer (see Figure 1). First, ImRs promotes autobiographical reasoning. As a result of the memory reappraisal, patients make more benign self-event connections and possibly construct a redemptive story in which the aversive experience has a positive outcome. Because redemption involves positive affective shifts $(35,37)$, the rescripted memories may acquire a more positive emotional tone. At the same time, the new selfevent connections, actively intervening in the memories, and imaginatively satisfying previously unmet needs may increase the sense of agency associated with these memories. Arntz and Weertman's (1) protocol explicitly incorporates need fulfillment and patients often employ memory intervention strategies which satisfy the needs they express in ImRs, including the need for autonomy [see (11)]. The increased sense of agency and mastery may contribute to recovery, as demonstrated by evidence that change in mastery of nightmare content mediated the efficacy of ImRs on nightmare frequency and distress (47). Second, since autobiographical reasoning may influence goal setting (48) and creating redemptive stories may lead to positive behavior change [see (49)], ImRs may also help patients set more realistic goals and abandon behaviors which helped maintain their symptoms (e.g., avoidance). This is why, as Brewin et al. (50) found, behavior change may occur spontaneously after ImRs. Third, changes in goals and in memories' meaning and affect may contribute to personality trait changes. Specifically, we propose that ImRs may contribute to reduced neuroticism and increased extraversion. This is because neuroticism is associated with negative affect (51), avoidance motivation (52), and more negative life chapters (53), whereas extraversion is associated with the opposite outcomes.

Personality changes may be amplified as individuals link the rescripted memories to other memories, modify further goals, and try to re-establish a sense of coherence in their life story and a sense of self-continuity and unity. They may, for example, reappraise other memories or new experiences in light of the rescripted memories' new meanings. Ultimately, these changes may influence the ways in which the rescripted memories - and potentially similar memories - exert their self and directive functions (36). In terms of the self-function, the new self-event connections could be incorporated into new or existing working selves which comprise more adaptive goals and self-images. The activation of these working selves following memory retrieval may then contribute to the rescripted memories' modified directive function as it elicits not only more positive self-images and self-evaluations, but also more positive affect and adaptive behaviors. In the long run, the frequent activation of the benign working selves may contribute to enhanced self-esteem and well-being [see (36)]. Taken together, these changes may account for the effectiveness of ImRs in reducing memory/imagery distress, targeting symptoms (3), and enhancing self-evaluations (8).

\section{CONCLUSION}

Our model (36) takes a novel approach as it utilizes the narrative identity literature to elucidate the mechanisms of change in interventions like ImRs. Of course, this model has limitations. For example, Kazdin (54) argues that mechanisms of change need to be studied as mediators of treatment effects and the temporal precedence of mediator changes over outcome changes needs to be established. If ImRs is effective at reducing symptoms after 1 or few sessions (3) and personality traits require weeks or months to change (42), then trait changes may be simple treatment outcomes rather than mechanisms and may play a greater role in preventing relapse. Furthermore, we acknowledge that the empirical testing of our model is likely to be complex and that it may be difficult to establish whether even changes in the life story and goals are mechanisms or treatment effects. Following Kazdin's approach for assessing change would require multiple personality and symptom assessments, as well as long follow-ups, in order to understand the temporal sequence of the changes experienced by patients and whether the variables we have identified in our model mediate treatment effects. As the study of mechanisms in psychotherapy remains under scrutiny and there is a growing number of proposals on how to assess them [see (55-58)], determining the best way to test our model will also require further developments and consensus in this field. 
Despite these limitations, we think that our model advances our understanding of ImRs. As the need to understand mechanisms of change in therapy becomes more pressing, we believe that it is essential for research into ImRs and similar interventions to recognize the complexity of personality. This may enable us to maximize the effectiveness of these interventions, delineate better when to use them, and identify who might relapse. Psychotherapy is an arena in which individuals make sense of their experiences and construct a coherent narrative identity (44, 59, 60). It may be time to recognize that through interventions like ImRs patients may rewrite their life story, not just isolated memories. The growing research on

\section{REFERENCES}

1. Arntz A, Weertman A. Treatment of childhood memories: theory and practice. Behav Res Ther. (1999) 37:715-40. doi: 10.1016/s0005-7967(98)00173-9

2. Wild J, Hackmann A, Clark DM. Rescripting early memories linked to negative images in social phobia: a pilot study. Behav Ther. (2008) 39:47-56. doi: 10.1016/j.beth.2007.04.003

3. Morina N, Lancee J, Arntz A. Imagery rescripting as a clinical intervention for aversive memories: a meta-analysis. J Behav Ther Exp Psychiatry. (2017) 55:6-15. doi: 10.1016/j.jbtep.2016.11.003

4. Dugué R, Renner F, Austermann M, Tuschen-Caffier B, Jacob GA. Imagery rescripting in individuals with binge-eating behavior: an experimental proofof-concept study. Int J Eat Disord. (2019) 52:183-8. doi: 10.1002/eat.22995

5. Fink J, Pflugradt E, Stierle C, Exner C. Changing disgust through imagery rescripting and cognitive reappraisal in contamination-based obsessive-compulsive disorder. J Anxiety Disord. (2018) 54:36-48. doi: 10.1016/j.janxdis.2018.01.002

6. Maloney G, Koh G, Roberts S, Pittenger C. Imagery rescripting as an adjunct clinical intervention for obsessive compulsive disorder. J Anxiety Disord. (2019) 66:102110. doi: 10.1016/j.janxdis.2019.102110

7. Tenore K, Basile B, Cosentino T, De Sanctis B, Fadda S, Femia G, et al. Imagery rescripting on guilt-inducing memories in OCD: a single case series study. Front Psychiatry. (2020) 11:543806. doi: 10.3389/fpsyt.2020.543806

8. Moritz S, Ahlf-Schumacher J, Hottenrott B, Peter U, Franck S, Schnell T, et al. We cannot change the past, but we can change its meaning. A randomized controlled trial on the effects of self-help imagery rescripting on depression. Behav Res Ther. (2018) 104:74-83. doi: 10.1016/j.brat.2018.02.007

9. Yamada F, Hiramatsu Y, Murata T, Seki Y, Yokoo M, Noguchi R, et al. Exploratory study of imagery rescripting without focusing on early traumatic memories for major depressive disorder. Psychol Psychother. (2018) 91:34562. doi: $10.1111 /$ papt.12164

10. Nilsson J-E, Knutsson J, Jalamo B-S, Lundh L-G. Imagery rescripting of early memories in health anxiety disorder: a feasibility and nonrandomized pilot study. J Behav Ther Exp Psychiatry. (2019) 65:101491. doi: 10.1016/j.jbtep.2019.101491

11. Romano M, Hudd T, Huppert JD, Reimer SG, Moscovitch DA. Imagery rescripting of painful memories in social anxiety disorder: a qualitative analysis of needs fulfillment and memory updating. Cogn Ther Res. (2020) 45:902-17. doi: 10.1007/s10608-020-10149-6

12. Romano M, Moscovitch DA, Huppert JD, Reimer SG, Moscovitch M. The effects of imagery rescripting on memory outcomes in social anxiety disorder. J Anxiety Disord. (2020) 69:102169. doi: 10.1016/j.janxdis.2019.102169

13. Schaitz C, Kroener J, Maier A, Connemann BJ, Sosic-Vasic Z. Short imagery rescripting intervention to treat emotionally dysregulated behavior in borderline personality disorder: an exploratory study. Front Psychiatry. (2020) 11:425. doi: 10.3389/fpsyt.2020.00425 autobiographical memory, narrative identity, and mental health may provide some of the best tools for understanding this rewriting process.

\section{AUTHOR CONTRIBUTIONS}

All authors listed have made a substantial, direct, and intellectual contribution to the work and approved it for publication.

\section{FUNDING}

The open access publication fee was provided by the University of Southampton.

14. Taylor CDJ, Bee PE, Kelly J, Emsley R, Haddock G. iMAgery focused psychological therapy for persecutory delusions in PSychosis (iMAPS): a multiple baseline experimental case series. Behav Cogn Psychother. (2020) 48:530-45. doi: 10.1017/S1352465820000168

15. Paulik G, Steel C, Arntz A. Imagery rescripting for the treatment of trauma in voice hearers: a case series. Behav Cogn Psychother. (2019) 47:709-25. doi: 10.1017/S1352465819000237

16. Arntz A. Imagery rescripting for personality disorders. Cogn Behav Pract. (2011) 18:466-81. doi: 10.1016/j.cbpra.2011.04.006

17. Dibbets P, Arntz A. Imagery rescripting: is incorporation of the most aversive scenes necessary? Memory. (2016) 24:683-95. doi: 10.1080/09658211.2015.1043307

18. Dibbets $\mathrm{P}$, Poort H, Arntz A. Adding imagery rescripting during extinction leads to less ABA renewal. J Behav Ther Exp Psychiatry. (2012) 43:614-24. doi: 10.1016/j.jbtep.2011.08.006

19. Mancini A, Mancini F. Rescripting memory, redefining the self: a metaemotional perspective on the hypothesized mechanism(s) of imagery rescripting. Front Psychol. (2018) 9:581. doi: 10.3389/fpsyg.2018.00581

20. Çili S, Pettit S, Stopa L. Impact of imagery rescripting on adverse self-defining memories and post-recall working selves in a nonclinical sample: a pilot study. Cogn Behav Ther. (2017) 46:75-89. doi: 10.1080/16506073.2016.1212396

21. Conway MA, Pleydell-Pearce CW. The construction of autobiographical memories in the self-memory system. Psychol Rev. (2000) 107:261-88. doi: 10.1037/0033-295x.107.2.261

22. Conway MA, Singer JA, Tagini A. The self and autobiographical memory: correspondence and coherence. Soc Cogn. (2004) 22:491-529. doi: 10.1521 /soco.22.5.491.50768

23. Brewin CR. Understanding cognitive behaviour therapy: a retrieval competition account. Behav Res Ther. (2006) 44:765-84. doi: 10.1016/j.brat.2006.02.005

24. Brewin CR, Gregory JD, Lipton M, Burgess N. Intrusive images in psychological disorders: characteristics, neural mechanisms, and treatment implications. Psychol Rev. (2010) 117:210-32. doi: 10.1037/a00 18113

25. Norton AR, Abbott MJ. The efficacy of imagery rescripting compared to cognitive restructuring for social anxiety disorder. J Anxiety Disord. (2016) 40:18-28. doi: 10.1016/j.janxdis.2016.03.009

26. Bluck S, Alea N, Habermas T, Rubin DC. A tale of three functions: the self-reported uses of autobiographical memory. Soc Cogn. (2005) 23:91-117. doi: $10.1521 /$ soco.23.1.91.59198

27. Pillemer DB. Remembering personal circumstances: a functional analysis. In: Winograd E, Neisser U, editors. Affect and Accuracy in Recall: Studies of "Flashbulb" Memories. Vol. 4. New York, NY: Cambridge University Press (1992). p. 236-64.

28. Pillemer DB. Directive functions of autobiographical memory: the guiding power of the specific episode. Memory. (2003) 11:193-202. doi: $10.1080 / 741938208$ 
29. Burnell R, Rasmussen AS, Garry M. Negative memories serve functions in both adaptive and maladaptive ways. Memory. (2020) 28:494-505. doi: 10.1080/09658211.2020.1737133

30. Ricarte JJ, Navarro B, Latorre JM, Ros L, Watkins E. Age and gender differences in emotion regulation strategies: autobiographical memory, rumination, problem solving and distraction. Span J Psychol. (2016) 19:1-9. doi: $10.1017 /$ sjp. 2016.46

31. McAdams DP. What do we know when we know a person? J Pers. (1995) 63:365-96. doi: 10.1111/j.1467-6494.1995.tb00500.x

32. McAdams DP. The psychology of life stories. Rev Gen Psychol. (2001) 5:10022. doi: 10.1037/1089-2680.5.2.100

33. McAdams DP. The psychological self as actor, agent, and author. Perspect Psychol Sci. (2013) 8:272-95. doi: 10.1177/1745691612464657

34. Singer JA, Salovey P. The Remembered Self: Emotion and Memory in Personality. New York, NY: The Free Press (1993).

35. Adler JM, Lodi-Smith J, Philippe FL, Houle I. The incremental validity of narrative identity in predicting well-being: a review of the field and recommendations for the future. Pers Soc Psychol Rev. (2016) 20:142-75. doi: $10.1177 / 1088868315585068$

36. Çili S, Stopa L. Autobiographical Memory and the Self: Relationship and Implications for Cognitive-Behavioural Therapy. London: Routledge (2019).

37. McLean KC, Syed M, Pasupathi M, Adler JM, Dunlop WL, Drustrup D, et al. The empirical structure of narrative identity: the initial Big Three. J Pers Soc Psychol. (2020) 119:920-44. doi: 10.1037/pspp0000247

38. Gregory B, Peters L. Changes in the self during cognitive behavioural therapy for social anxiety disorder: a systematic review. Clin Psychol Rev. (2017) 52:1-18. doi: 10.1016/j.cpr.2016.1 1.008

39. Gregory B, Wong QJJ, Marker CD, Peters L. Maladaptive self-beliefs during cognitive behavioural therapy for social anxiety disorder: a test of temporal precedence. Cogn Ther Res. (2018) 42:261-72. doi: 10.1007/s10608-017-9 882-5

40. Allemand M, Flückiger C. Changing personality traits: some considerations from psychotherapy process-outcome research for intervention efforts on intentional personality change. J Psychother Integr. (2017) 27:476-94. doi: 10.1037/int000 0094

41. Hengartner MP, Von Wyl A, Haldimann BH, Yamanaka-Altenstein M. Personality traits and psychopathology over the course of six months of outpatient psychotherapy: a prospective observational study. Front Psychol. (2020) 11:174. doi: 10.3389/fpsyg.2020.00174

42. Roberts BW, Luo J, Briley DA, Chow PI, Su R, Hill PL, et al. A systematic review of personality trait change through intervention. Psychol Bull. (2017) 143:117-41. doi: 10.1037/bul0000088

43. Zinbarg RE, Uliaszek AA, Adler JM. The role of personality in psychotherapy for anxiety and depression. J Pers. (2008) 76:1649-88. doi: 10.1111/j.1467-6494.2008.00534.x

44. Adler JM. Living into the story: agency and coherence in a longitudinal study of narrative identity development and mental health over the course of psychotherapy. J Pers Soc Psychol. (2012) 102:367-89. doi: 10.1037/a0025289

45. Lind M, Jørgensen CR, Heinskou T, Simonsen S, Bøye R, Thomsen DK. Patients with borderline personality disorder show increased agency in life stories after 12 months of psychotherapy. Psychotherapy. (2019) 56:274-84. doi: $10.1037 /$ pst0000184

46. Quitasol MN, Fournier MA, Di Domenico SI, Bagby RM, Quilty LC. Changes in psychological need fulfillment over the course of treatment for major depressive disorder. $J$ Soc Clin Psychol. (2018) 37:381-404. doi: 10.1521/jscp.2018.37. 5.381
47. Kunze AE, Lancee J, Morina N, Kindt M, Arntz A. Mediators of change in imagery rescripting and imaginal exposure for nightmares: evidence from a randomized wait-list controlled trial. Behav Ther. (2019) 50:978-93. doi: 10.1016/j.beth.2019.03.003

48. Bluck S, Habermas T. The life story schema. Motiv Emot. (2000) 24:121-47. doi: 10.1023/A:1005615331901

49. Dunlop WL, Tracy JL. Sobering stories: narratives of self-redemption predict behavioral change and improved health among recovering alcoholics. J Pers Soc Psychol. (2013) 104:576-90. doi: 10.1037/a0031185

50. Brewin CR, Wheatley J, Patel T, Fearon P, Hackmann A, Wells A, et al. Imagery rescripting as a brief stand-alone treatment for depressed patients with intrusive memories. Behav Res Ther. (2009) 47:569-76. doi: 10.1016/j.brat.2009.03.008

51. Steel P, Schmidt J, Shultz J. Refining the relationship between personality and subjective well-being. Psychol Bull. (2008) 134:138-61. doi: 10.1037/0033-2909.134. 1.138

52. Elliot AJ, Thrash TM. Approach-avoidance motivation in personality: approach and avoidance temperaments and goals. $J$ Pers Soc Psychol. (2002) 82:804-18. doi: 10.1037/0022-3514.82. 5.804

53. Thomsen DK, Olesen MH, Schnieber A, Tønnesvang J. The emotional content of life stories: positivity bias and relation to personality. Cogn Emot. (2014) 28:260-77. doi: 10.1080/02699931.2013.815155

54. Kazdin AE. Understanding how and why psychotherapy leads to change. Psychother Res. (2009) 19:418-28. doi: 10.1080/10503300802448899

55. Carey TA, Griffiths R, Dixon JE, Hines S. Identifying functional mechanisms in psychotherapy: a scoping systematic review. Front Psychiatry. (2020) 11:291. doi: 10.3389/fpsyt.2020.00291

56. Hayes AM, Andrews LA, A. complex systems approach to the study of change in psychotherapy. BMC Med. (2020) 18:197. doi: 10.1186/s12916-020-01662-2

57. Mansell W, Huddy V. Why do we need computational models of psychological change and recovery, and how should they be designed and tested? Front Psychiatry. (2020) 11:624. doi: 10.3389/fpsyt.2020.00624

58. Watkins ER, Newbold A. Factorial designs help to understand how psychological therapy works. Front Psychiatry. (2020) 11:429. doi: 10.3389/fpsyt.2020.00429

59. McAdams DP, McLean KC. Narrative identity. Curr Dir Psychol Sci. (2013) 22:233-8. doi: 10.1177/0963721413475622

60. Singer JA. Personality and Psychotherapy: Treating the Whole Person. New York, NY: The Guilford Press (2005).

Conflict of Interest: The authors declare that the research was conducted in the absence of any commercial or financial relationships that could be construed as a potential conflict of interest.

Publisher's Note: All claims expressed in this article are solely those of the authors and do not necessarily represent those of their affiliated organizations, or those of the publisher, the editors and the reviewers. Any product that may be evaluated in this article, or claim that may be made by its manufacturer, is not guaranteed or endorsed by the publisher.

Copyright (C) 2021 Çili and Stopa. This is an open-access article distributed under the terms of the Creative Commons Attribution License (CC BY). The use, distribution or reproduction in other forums is permitted, provided the original author(s) and the copyright owner(s) are credited and that the original publication in this journal is cited, in accordance with accepted academic practice. No use, distribution or reproduction is permitted which does not comply with these terms. 